\title{
Evidence for a Flux Rope driven EUV wave and CME: Comparison with the Piston Shock Model
}

\author{
C. R. Foley ${ }^{1}$, L. K. Harra ${ }^{1}$, S. A. Matthews ${ }^{1}$, J. L. Culhane ${ }^{1}$, and R. Kitai ${ }^{2}$ \\ ${ }^{1}$ Mullard Space Science Laboratory, Department of Space and Climate Physics, Holmbury St Mary, Dorking, Surrey, \\ RH5 6NT, UK \\ 2 Kwasan and Hida Observatories, Kyoto University, Yamashina, Kyoto 607-8471, Japan
}

Received 4 October 2002 / Accepted 5 December 2002

\begin{abstract}
This paper examines the relationship between a coronal wave, filament eruption, flare and Coronal Mass Ejection (CME) which occurred on 2001, April 10th. We study the pre-flare activity which includes the eruption of a filament and a coronal wave. A large X-ray flare and a CME follow. We discuss how these phenomena are related and compare our results to recent models. These are found to be largely consistent with the numerical simulations of a flux rope driven CME as presented recently in Chen et al. (2002).
\end{abstract}

Key words. Sun: coronal mass ejections (CMEs) - Sun: corona - Sun: filaments

\section{Introduction}

Coronal waves have been identified for a number of events in the EUV images obtained by the EUV Imaging Telescope (EIT) instrument on SOHO (e.g. Thompson et al. 1999). These wave like motions which evolve from central locations are sometimes referred to as EIT waves. Before the SOHO mission waves had been identified back in the early fifties by Moreton in full sun $\mathrm{H} \alpha$ images (e.g. Moreton 1961). However, these two types of waves possess different characteristics, and this has resulted in a lively debate regarding the origins and relationship between these types of wave. Uchida (1968) proposed that Moreton waves are the skirt of a fast-mode wave in the corona caused by a flare. When EIT coronal waves were first observed it was suggested that they may be the coronal counterpart of Uchida's Moreton waves. Since then other suggestions have been made including a fast CME driving a shock (Cliver et al. 1999), or that they are due to field lines opening during the CME onset (Delannee \& Aulanier 1999).

Recent numerical simulations (Chen et al. 2002) have investigated the relationship between Moreton waves and coronal waves. The modeling of an erupting flux rope revealed that in front of the expanding flux rope, a piston driven shock forms along the envelope of the expanding CME. The legs of this shock which sweep the solar surface as it propagates, produces the aforementioned Moreton waves. Behind this, a slower-moving wave-like structure was resolved in the simulations, being heralded as most likely responsible for the coronal EUV waves.

Send offprint requests to: C. Foley, e-mail: carl.foley@mssl.ucl.ac.uk
Recent work by Biesecker et al. (2002) has looked at the correlations between EIT waves, flares and CMEs for a sample of 173 EIT waves. After accounting for observational biases, they find that EIT waves are accompanied by CMEs in all cases, but that the converse is not always true. In addition they also found that not every EIT wave is accompanied by a flare. In this paper, we examine the relationship between a coronal wave which was accompanied by an X2.3 flare. The event occurred on the 10th April 2001. Various aspects of this event have been studied in detail so far. These include spectroscopic observations of a flux rope erupting (Foley et al. 2001; Pike \& Mason 2002) and a study of the spatial distribution of the hard $\mathrm{X}$-ray emission and the $\mathrm{H} \alpha$ kernels (see Asai et al. 2002).

This paper examines the events which occurred on 2001, April 10th in AR 9415 from 20 min before the flare impulsive phase to the propagation of the CME. The purpose is to determine the relationship between an erupting flux rope, coronal waves, and a CME.

In the following sections we describe the observations of the flare and CME, the associated EUV waves which were observed with both TRACE and EIT. We demonstrate that the flux rope evolves to form the core of the CME. We then go on to discuss the observational features in the context of the flux rope CME numerical model of Chen et al. (2002).

\section{Instrumentation}

Full disc images of the Sun were also obtained with the SOHO EUV imaging Telescope (EIT) in a bandpass of $195 \AA$. These images are dominated by emission from Fe XII which forms at temperatures in the region of 1.3 MK. These EIT images 


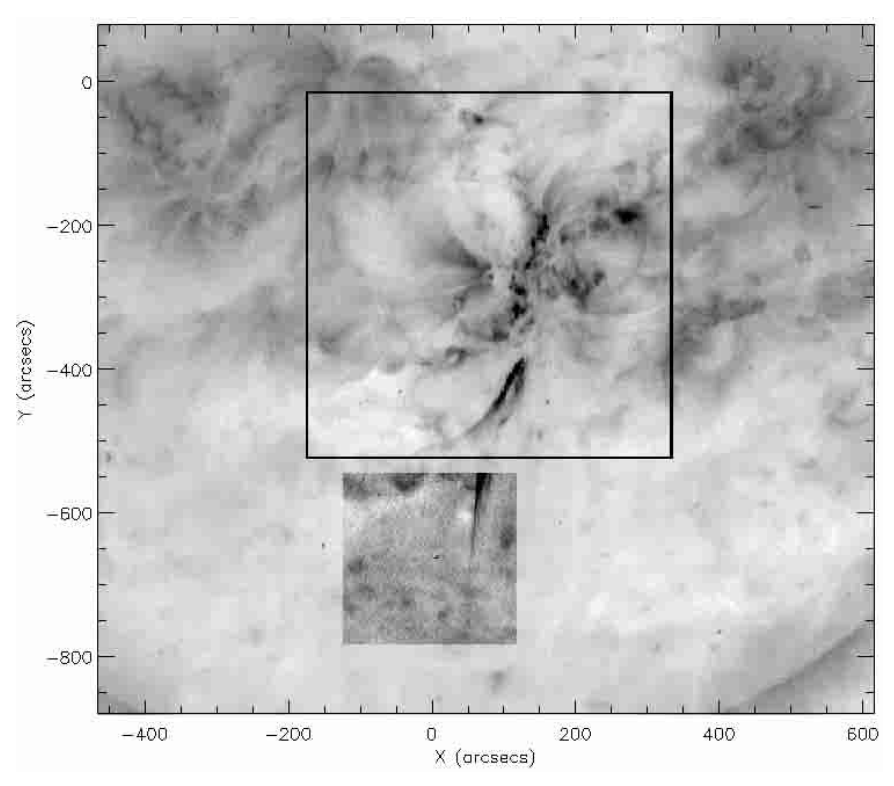

Fig. 1. The EIT $195 \AA$ image obtained around the onset of the X2.3 flare which occurred on 2001, April 10th. The TRACE field of view is indicated with the inset TRACE observation at which is positioned above the inset CDS field of view. The transit of the flux rope from the TRACE fov is clear as streak in this OV image.

were obtained with a resolution of 2.5 arcsec, and a cadence of $12 \mathrm{~min}$. Partial frame images $(512 \times 512$ arcsec $)$ were obtained of active region 9415 with the $171 \AA$ filter of TRACE (Handy et al. 1999). These TRACE $171 \AA$ images are dominated by Fe IX which forms at around $0.95 \mathrm{MK}$. These images were obtained with a count rate dependent exposure control which produced exposures from around 23 to $4 \mathrm{~s}$, and were rebinned on the CCD to 4 arcsec resolution from a native resolution of 1 arcsec. Coronagraph images of the associated CME were obtained with the SOHO Large Angle Solar Coronagraph (LASCO - Brueckner et al. 1995). These images are recorded with a cadence of approximately $24 \mathrm{~min}$ in its C2 band $\left(2-6 R_{\odot}\right)$ and 36 mins in its $\mathrm{C} 3$ band $\left(4-30 R_{\odot}\right.$ ). Full Sun soft X-ray flux in the $0.5-4 \AA$ and $1-8 \AA$ passband were recorded with the GOES 10 spacecraft.

\section{Observations}

In this section we describe the pre-flare observations of Active Region 9415 on 2001, 10th April, the X2.3 flare which peaked at 05:26 UT, and the CME which was first observed with LASCO at 05:30UT.

The key characteristics of the event are that AR 9415 was observed to expand gradualy $\left(\sim 100 \mathrm{~km} \mathrm{~s}^{-1}\right)$ some twenty or so minutes before the flares impulsive phase ( 05:10 UT). This expansion was characterised by a dimming observed in the EIT images at 04:56 UT. An EUV wave was identified at 05:08 UT in the EIT $195 \AA$ images, which was also recorded in the TRACE $171 \AA$ images. This was found to be and located just in front of the flux rope.

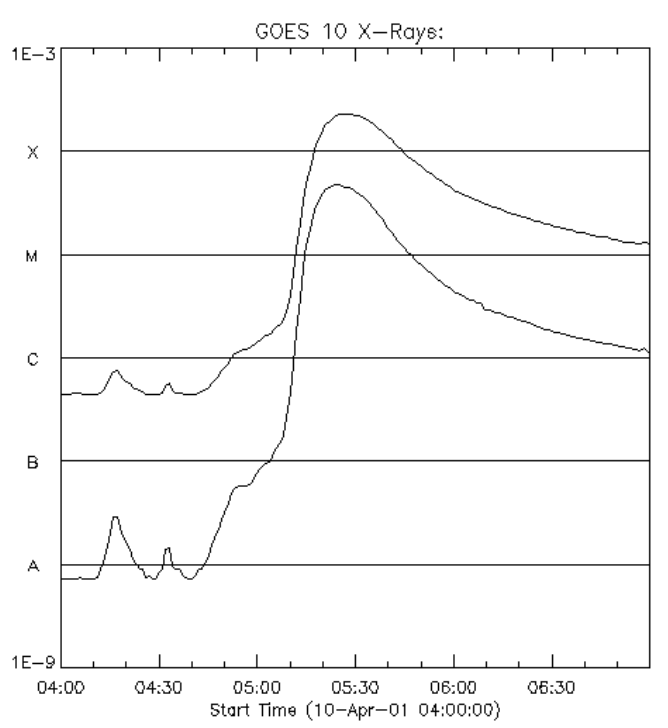

Fig. 2. GOES 10 full Sun soft X-ray flux in the $0.5-4 \AA$ and $1-8 \AA$ passband. The pre-flare brightening can be seen as a shoulder in the light curve commencing at around 04:40 UT, while the impulsive phase occurs around 05:10 UT.

\subsection{Pre-flare}

Active region NOAA 9415 was located close to the centre of the Sun on the 2001, April 10th. The importance of this is that any associated CME may be Earth directed and more likely to produce geomagnetic disturbances. The central location also allows the evolution of the active region and the photospheric field to be clearly evaluated. Over the days leading up to the 10th, the photospheric field was observed to rotate strongly, most probably building up helicity and shear within active region 9415 . On the morning of the 10th this was most clearly apparent within the SXT image which displayed the region as coiled, and sigmoidal (see Fig. 2, Foley et al. 2001). An EIT image with an inset TRACE image obtained at around the same time (during which the flux rope was in mid-eruption) of 05:08 UT are displayed in Fig. 1. The flux rope can be seen clearly in the TRACE image located at around $\left(50^{\prime \prime},-450^{\prime \prime}\right)$.

Starting at $\sim 04: 40$ UT a pre-flare brightening was observed to occur in the GOES light curves (see Fig. 2). This gradual start to a flare is usually associated with filament eruptions, and believed to be due to the relatively slow stressing of the field before the flares impulsive phase. This was confirmed in this case by the $\mathrm{H} \alpha$ observations provided by the HIDA Observatory, (see Fig. 3). These reveal a filament structure to be lifting towards the south of the active region.

On inspection of the TRACE images it appears that the pre-flare brightening observed in GOES was also observed in the TRACE images (see Fig. 4) around the location of an absorption feature, consistent with the filament location recorded with the HIDA observations. Filament material generally manifests itself as absorption features in EUV images due to the high optical depth of the material. The light curve (see toppanel Fig. 4) was formed from the region around the brightening. A background threshold, was used to exclude bad data associated with the spacecraft passage of the South Atlantic 


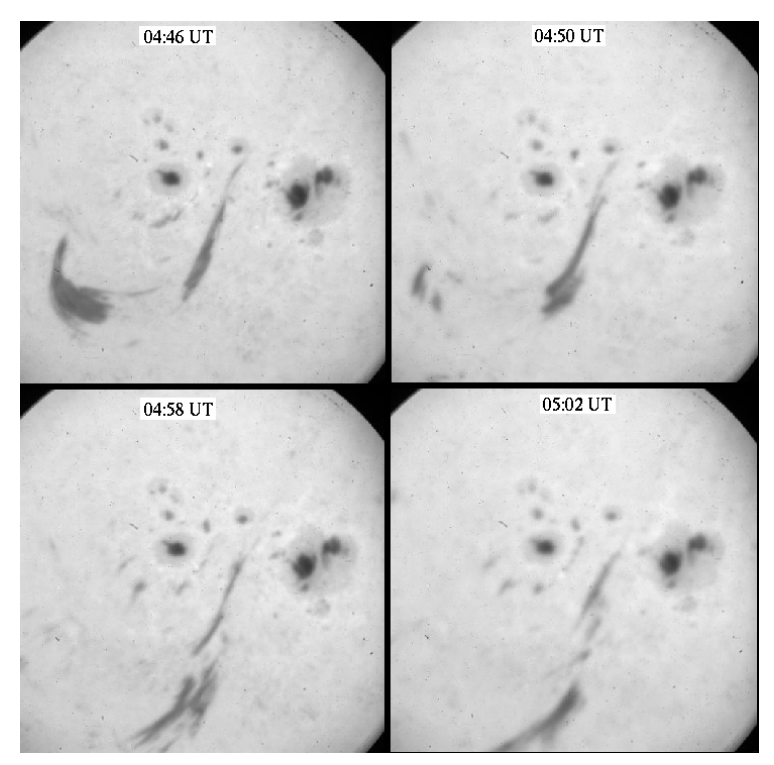

Fig. 3. DST H-alpha images of the filament eruption recorded by the HIDA observatory. The velocity of the flux rope was determined to be of the $\sim 100 \mathrm{~km} \mathrm{~s}^{-1}$ consistent with that found later in the TRACE images.

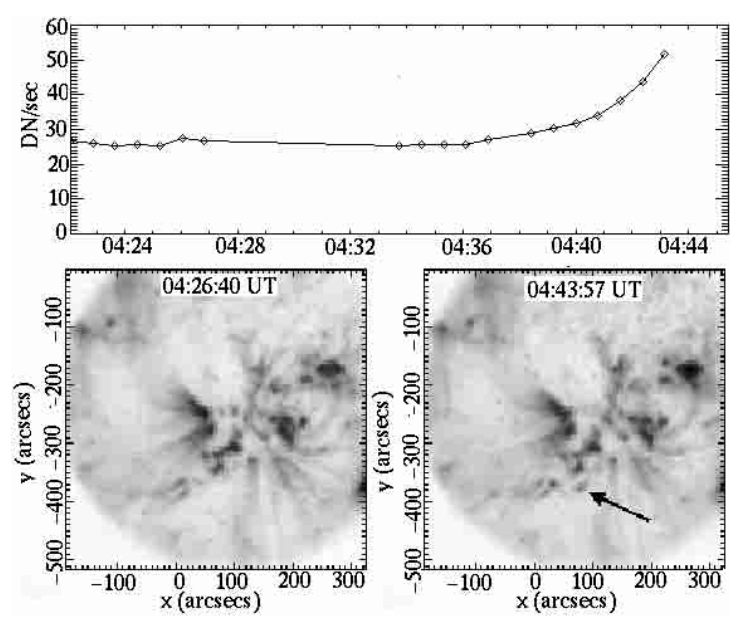

Fig. 4. The location of the brightening in the TRACE $171 \AA$ images (indicated by arrow bottom right), along with a light curve formed for the region (top). The vertical lines on the light curve indicate the image time.

Anomaly. Although, the flux rope was observed to move slowly outwards no TRACE images were taken between 04:47 UT and 04:59 UT. In the images after this the flux rope was close to the bottom of the TRACE field of view, and the eruption of the flux rope was observed to continue towards the south of the active region. In Fig. 1, we display a TRACE image obtained at this time inset into an EIT $195 \AA$ image, along with an inset CDS OV image. The OV image is formed by integrating the OV $629.7 \AA$ emission line.

To the right (west) of the active region material was observed to expand outwards. The speed for this expansion was estimated by tracking features in the running difference images (see Fig. 5). This method implied that the front was moving at a speed of $\sim 80-120 \mathrm{~km} \mathrm{~s}^{-1}$. Structures to the east (left) of
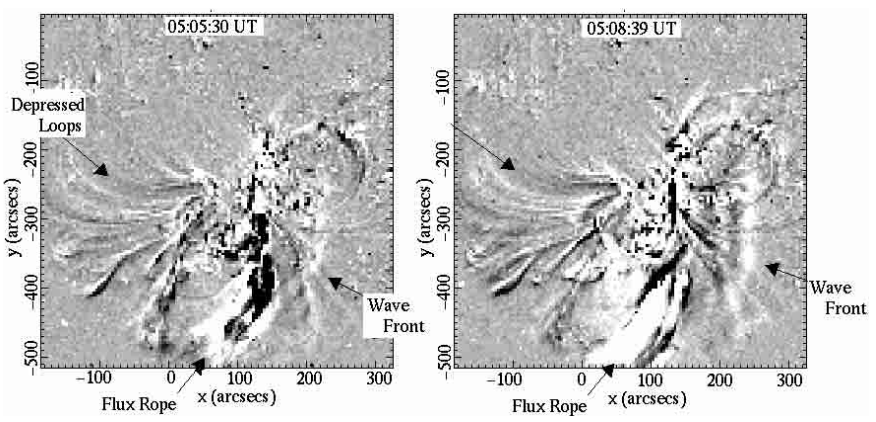

Fig. 5. TRACE difference images are displayed at 05:06 UT on the left and 05:08 UT on the right. These reveal the apparent motion and brightening of material from frame to frame.

the AR appeared to be depressed, almost as if being deflected by a similarly propagating front. It is not possible to determine whether a similar front is expanding to the south since its motion would be obscured by the flux rope, although it appears so since the front extends out of the field of view to the south. The velocity of the flux rope was determined in a similar fashion to be $\sim 130 \mathrm{~km} \mathrm{~s}^{-1}$.

We then investigated the EIT images obtained around the time of the flare for evidence of a wave. We processed all the images using the standard EIT calibration procedure EIT_PREP. In Fig. 6, we illustrate running difference images which have been derived simply by removal of the image obtained some twelve minutes earlier.

There is an impressive "EIT wavefront" associated with this event which can be seen to commence at 05:08UT, just before the flare's impulsive (or flash) phase which started at $\sim 05: 10$ UT (see Fig. 6). The speed of the front has been estimated from the speed with which the dimming region extends from one image to the next, in the running difference images. We have used this method since this is a clearly defined boundary associated with the front. The speeds obtained using this method (200-250 $\mathrm{km} \mathrm{s}^{-1}$ ) are similar although somewhat larger than the speeds determined from the TRACE data for the flux rope and part of the front $\left(100-130 \mathrm{~km} \mathrm{~s}^{-1}\right)$. This may be due to an acceleration occurring during the flares impulsive phase at 05:10UT. However, this is only part of the front which we observe in the EIT image due to the limited field of view of the TRACE images. The moving front which we observed in the TRACE images also appears in the same location in the EIT $195 \AA$ difference images (bottom left of Fig. 6, at $\left.\left[50^{\prime \prime},-450^{\prime \prime}\right]\right)$ confirming that we are looking at similar features. Also, this difference image demonstrates that the EUV wave preceded at or close to the erupting flux rope as was implied by the TRACE difference images (cf. Fig. 5). If we ignore any possible acceleration the origin of the wavefront observed in the EIT images would have to have an origin in the region of 04:50 UT, and earlier than that if infact the wavefront we observed experiences some form of acceleration over the observation. 

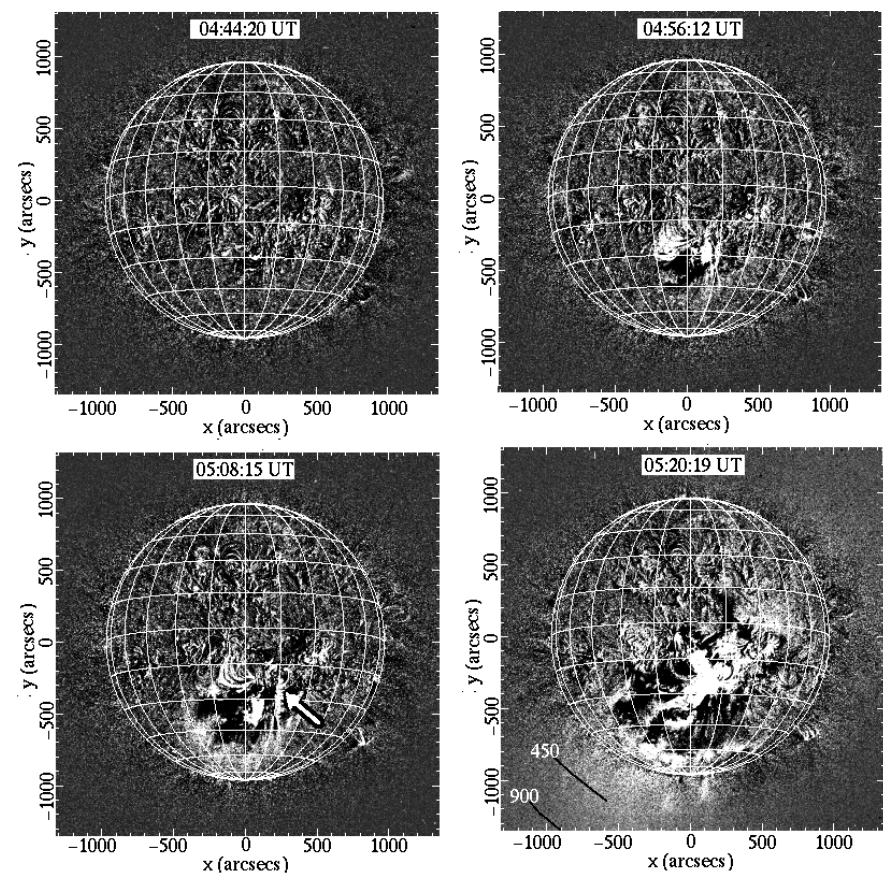

Fig. 6. EIT $195 \AA$ A running difference images of the Sun covering the period of the X2.3 flare. The dimming and bright front originally appears during the pre-flare phase and appears to expand only slightly (approx $180 \mathrm{~km} \mathrm{~s}^{-1}$ west and $80 \mathrm{~km} \mathrm{~s}^{-1}$ east) prior to the impulsive phase of the flare, after which the effects are broadly observed with the ejected material clearly visible above the limb. In the bottom left panel the location of the bright front which is observed in both TRACE and EIT is arrowed. In the bottom right panel the wave front appears as CME material above the south eastern limb (bottom left) over the entire height range. The distance travelled in the twelve minutes from the previous EIT frame is indicated by the black lines for speeds of $\sim 450$ and $900 \mathrm{~km} \mathrm{~s}^{-1}$. The average speed of the material over this timespan would have to be in the region of $900 \mathrm{~km} \mathrm{~s}^{-1}$ to explain the EIT emission above the south eastern limb.

\subsection{Flare}

The impulsive phase or rise phase of the 2001, April 10th flare was the subject of a paper by Foley et al. (2001). In this paper the bulk motion of the flux rope was examined in the TRACE images by a using a bootstrapping method to determine the progression of the flux rope through its field of view. This method revealed that the flux rope was being accelerated over the observed time interval. An investigation of the averaged Doppler velocities for the structure demonstrated that this acceleration could be separated into two acceleration phases, one which occurred at the impulsive phase of the flare, and a more gradual one which followed. The former of these was found to be consistent (Foley et al. 2002) with the motions of the flare ribbons which would be expected with standard flare models.

This work was questioned in Pike \& Mason (2002), who came up with an alternative description. This description identified components and tracked them through the CDS field of view. The problem with this alternative analysis however is that it considers components which are relatively dim and unimportant in comparison to the main body of the flux rope. This is demonstrated in Fig. 7 which illustrates the average Doppler
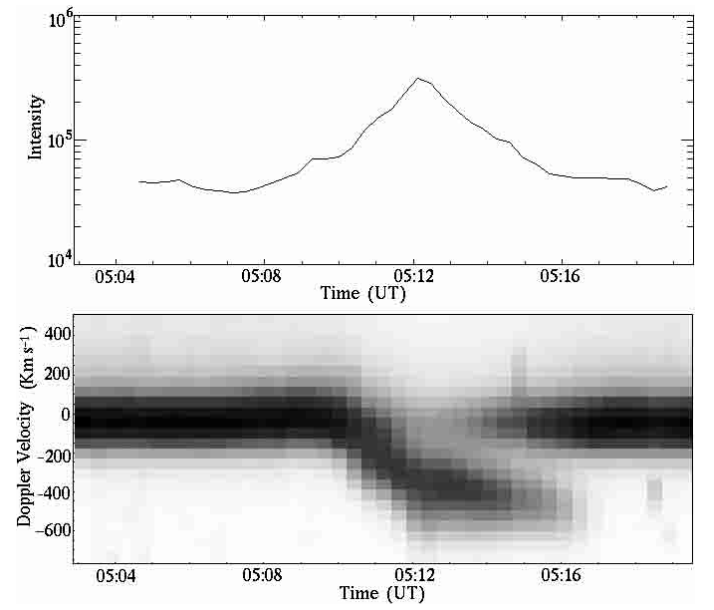

Fig. 7. Averaged CDS spectra verses time for the OV line. The line intensity is plotted verses time in the top plot. This has been used to normalise the intensity of the CDS spectra verses time in the plot below. The initial rapid acceleration and settling to a more gradual form is clear in the figure.

velocity (which has been normalized by the averaged intensity). It is clear from this plot that only a single component is required to explain the observations. Coupled with this there are no simultaneous observations of red and blue shifts in the CDS field of view which may be expected if we are indeed observing a structure with strong rotational motion and is contained entirely in the FOV. Instead what we see is relatively constrained distribution of velocities.

This is confirmed by considering the other observations which have been presented here. Most notably the EIT difference images illustrated in Fig. 6. The bottom two difference images represent the times 05:08 UT (left) and 05:20 UT (right), and span the impulsive phase of the flare. The dramatic difference is evident with the transit of the CME visible above the south eastern limb (bottom left) over the entire height range. This is of the order of a solar radii, which when compared to the previous image obtained taken some 12 min earlier there is no evidence for. For the material to travel a solar radii in $12 \mathrm{~min}$ its average speed would have to have been $\sim 900 \mathrm{~km} \mathrm{~s}^{-1}$. This suggests that the CME core had increased its speed from around the $130 \mathrm{~km} \mathrm{~s}^{-1}$, which was the velocity of the flux rope in the TRACE data prior to the flares impulsive phase, to a velocity in excess of $900 \mathrm{~km} \mathrm{~s}^{-1}$ to explain this EIT emission.

\section{3. $C M E$}

As mentioned in the previous section the speed of the CME core which entered the field of view of the LASCO is excess of $900 \mathrm{~km} \mathrm{~s}^{-1}$. The first evidence of the CME in the LASCO occurred in the image obtained at 05:30UT. This was the front of the CME " $F$ " and is indicated in Fig. 8. In this figure we have displayed the evolution of the front " $F$ " and core "C" of CME in the LASCO C2.

The evolution of these through the LASCO C2 and into the C3 field of view is displayed in Fig. 9. The CME doesn't appear to show any signs of acceleration or deceleration in the 


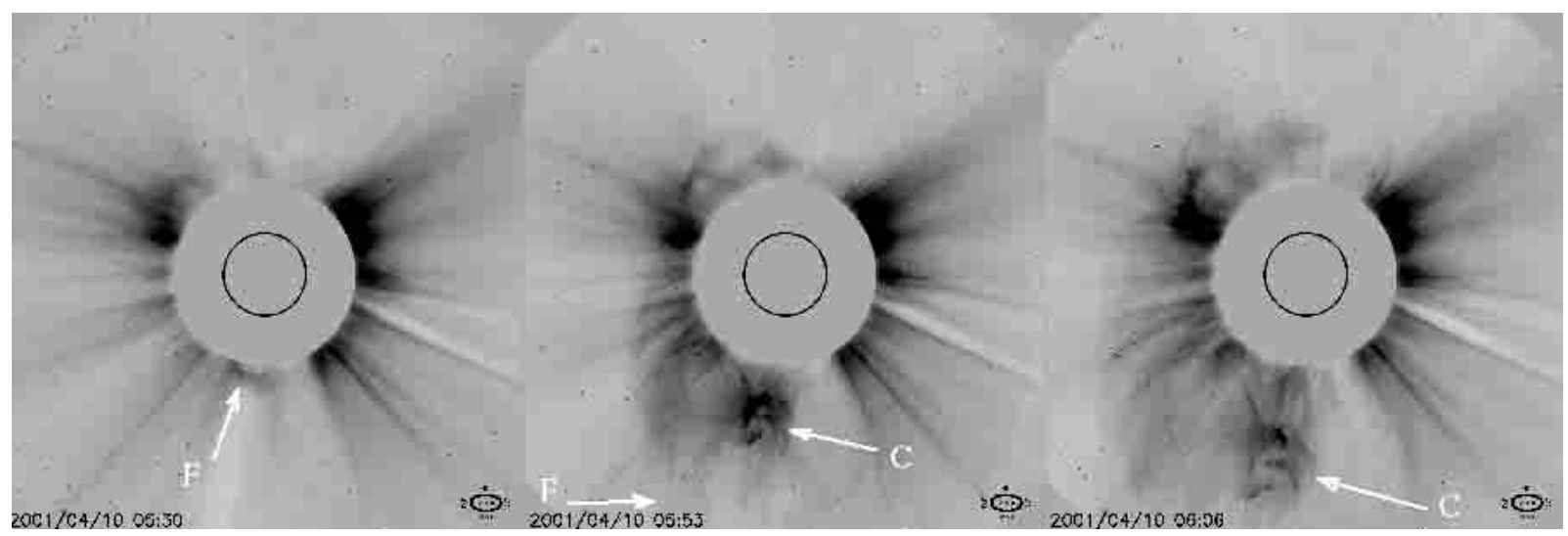

Fig. 8. Evolution of the 2001, April 10th CME as recorded by LASCO C2 (top to bottom) and C3. The CME front 'F' and core are indicated with arrows. These features were tracked into the $\mathrm{C} 3$ field of view to determine the speed of the CME in the plane of the sky.
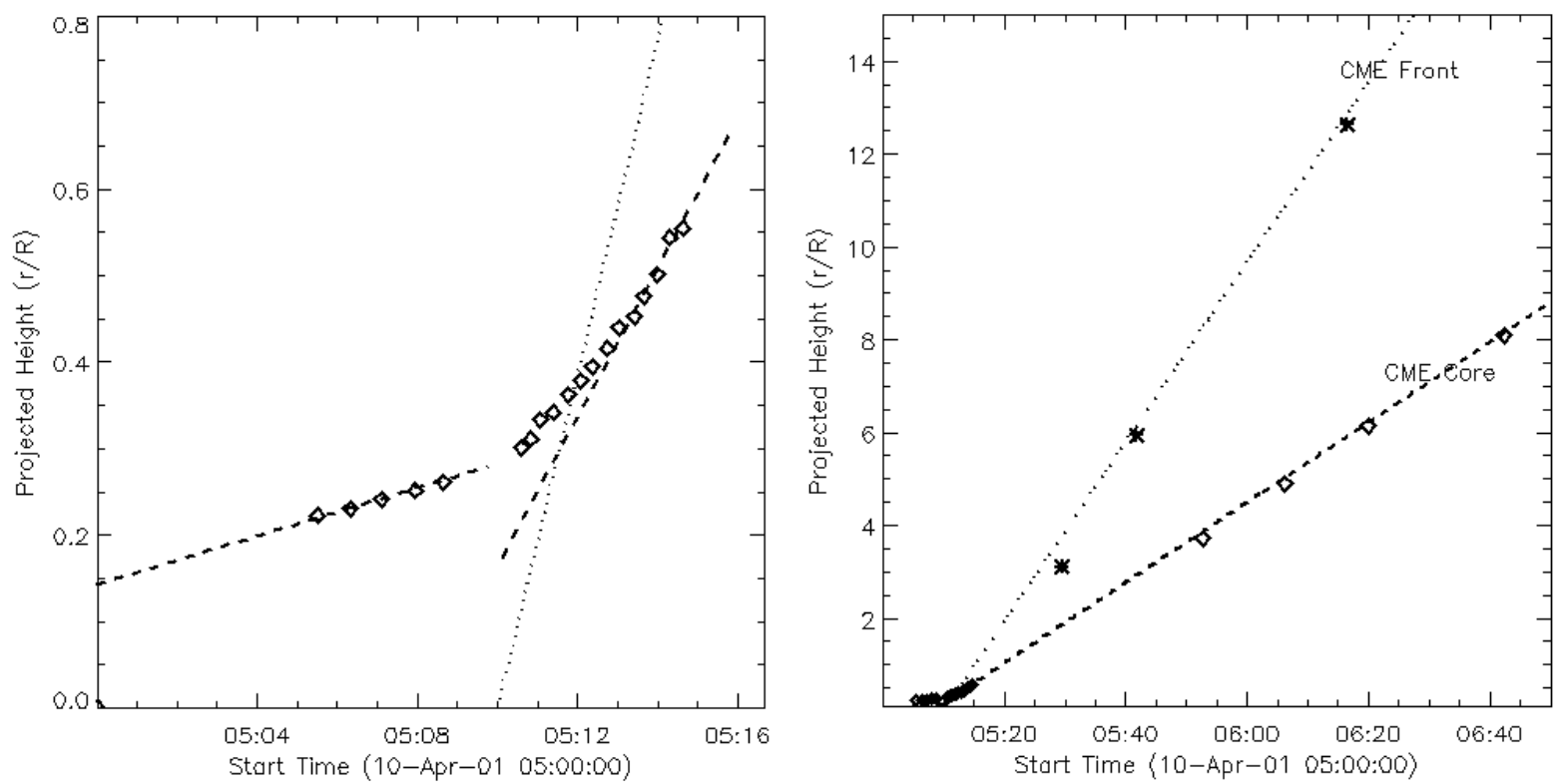

Fig. 9. The time height of the flux rope as observed by TRACE and LASCO C2 / C3. The LASCO features tracked are illustrated in Fig. 8. Diamonds represent the observations of the CME core by TRACE (below 1 Solar radii - left panel) and LASCO (above 2 solar radii - right panel) The front of the CME (which is only clearly observed in LASCO) are presented by the asterix's in the right hand panel. This CME front is traveling at twice the speed of the core (dotted line), and extrapolates back to the impulsive phase of the flare $\sim 05: 10$ UT.

LASCO (left panel of Fig. 9), and suggests that the CME was accelerated close to the Sun as was suggested in the previous section and indicated by the CME core observations by TRACE (right panel of Fig. 9). The front of the CME is traveling at $2200 \mathrm{~km} \mathrm{~s}^{-1}$, approximately twice as fast as the core. This front loses its identity in the LASCO C3, and is difficult to identify in subsequent images, presumably due to its intensity going below the limit of sensitivity for that Coronagraph. The core remains detectable although its morphology changes somewhat. Its velocity stays constantly $\sim 1200 \mathrm{~km} \mathrm{~s}^{-1}$.

\section{Discussion and conclusions}

We have presented observations of an X2.3 flare and associated CME which occurred on 2001, April 10th. The first manifestation of which was a general expansion of active region 9415. This initial expansion was most probably attributed to a strong build up of shear within the active region in the days prior to the event. The speed of the initial expansion and presumably the speed of the CME was in the region of $\sim 130 \mathrm{~km} \mathrm{~s}^{-1}$. The expansion occurred some twenty or so minutes before the flares impulsive phase. This is confirmed by a dimming surrounding the active region at 04:56 UT in the EIT difference images. No wave front was apparent in the EIT $195 \AA$ images at that time.

The EIT $195 \AA$ wave front was identified 05:08 UT, and was located just in front of the flux rope. At this time part of this wave front was also observed in the TRACE $171 \AA$ images at the same location. This had a velocity of $\sim 100 \mathrm{~km} \mathrm{~s}^{-1}$ in the TRACE images and most probably represented the initial expansion of Active Region 9415. In the next EIT image obtained at 05:20 UT, the CME was evident as enhanced emission above the south eastern limb. For this to have occurred the CME associated with the flux rope appears to experience a great acceleration probably around the impulsive phase of the 
flare, between 05:10 and 05:12 UT, as was originally suggested by the observations reported in Foley et al. (2001).

The speed of the front of the CME as determined from the LASCO data, is more than twice as fast as the core (flux rope) itself, being $\sim 2300 \mathrm{~km} \mathrm{~s}^{-1}$ and $\sim 1200 \mathrm{~km} \mathrm{~s}^{-1}$ respectfully. The origin of this front extrapolates back close to the impulsive phase of the flare while that of the core to some minutes earlier.

As mentioned in the introduction, numerical simulations of erupting flux ropes and the associated presence of wave like motions of the full Sun EUV observations were presented in Chen et al. (2002). In this work a rising flux rope produces a piston driven shock which straddles over the flux rope. This shock can travel at 2-3 times the speed of the flux rope and is the likely cause of the CME front which we observed to proceed infront of the flux rope. Particularly since its origin is close to the impulsive phase of the flare, when it is expected that energy is injected to the flux rope and CME around this time (Foley et al. 2001).

The propagation of this shock opens the field, and produces density enhancements which propagate across the face of the Sun, EUV waves. These are not a fast mode waves, but simply the effect of a density front which is formed by the continual opening of the field which naturally results from the advancing flux rope, behind which the dimming region appears as the plasma behind is evacuated.

It was also demonstrated by Chen et al. (2002), that the EUV wave typical travels at a terminal velocity of around $0.34\left(v_{\mathrm{A}}^{2}+v_{\mathrm{c}}^{2}\right)^{0.5}$ where $v_{\mathrm{A}}$ and $v_{\mathrm{c}}$ are the Alfven and sound speed respectfully. These speeds do not vary greatly in the quiet corona so it is unsurprising that the speed we determine for the EUV wave observed in EIT $195 \AA$ is close to the apparently canonical $250 \mathrm{~km} \mathrm{~s}^{-1}$ for EIT waves.

The main difference between our observations and those of Chen et al. (2002), is to respect to the speed of the expanding front. In our observations we found that this expansion (from the TRACE data) close to the active region was approximately half that which was found for the EIT data. This is counter to the discussion of the previous paragraph, where the Alfven and sound speeds would be expected to be higher close to the active region and thus the EUV wave be faster, and not traveling considerably more slowly. Although it is probably a result of the flux rope erupting at sub-sonic speeds prior to the impulsive phase of the flare. Apart from this discrepancy, our observations support the notion that many EUV waves could be the result of the shocks forming before erupting flux ropes. Flux ropes are central to many CME models and their presence within interplanetary CME's is well documented, so it may not be unreasonable for EUV waves and many CME to have this common element.
To conclude we believe our observations can be described in the context of the Chen et al. (2002), model. In this the initial expansion of the flux rope produces a dimming in the associated coronal EUV and soft X-ray images, this is followed shortly by a low coronal wave front which is produced by the skirt of the expanding CME, and is driven by a piston shock which straddles over the rising flux rope and ultimately drives the CME front. Our observations also support the view of Foley et al. (2001), that the flux rope and CME receive an additional energy during the impulsive phase of the flare, which explains the slower expansion close to the core of the active region. This may explain why flare associated CME tend to be brighter and faster than those without (e.g. see Andrews \& Howard 2001).

Acknowledgements. We would like to thank Douglas Biesecker for comments which improved the clarity of this work. We are grateful to the UK Particle Physics and Astronomy Research Council for financial support. We acknowledge the Solar UK Research Facility (SURF http://surfwww.mssl.ucl.ac.uk/surf/) for providing data for use in this publication. SOHO is a project of international cooperation between ESA and NASA. This research has made use of NASA's Astrophysics Data System Abstract Service.

\section{References}

Andrews, M. D., \& Howard, R. A. 2001, Space Sci. Rev., 95, 147

Asai, A., Yokoyama, T., Shimojo, M., et al. 2002, in Multi-Wavelength Observations of Coronal Structure and Dynamics, Yohkoh 10th Anniversary Meeting, ed. P. C. H. Martens, \& D. Cauffman, COSPAR Colloquia Ser., 13

Biesecker, D. A., Myers, D. C., Thompson, B. J., Hammer, D. M., \& Vourlidas, A. 2002, ApJ, 569, 1009

Brueckner, G. E., Howard, R. A., Koomen, M. J., et al. 1995, Sol. Phys., 162, 357

Chen, P. F., Wu, S. T., Shibata, K., \& Fang, C. 2002, ApJ, 572, L99

Cliver, E., Webb, D., \& Howard, R. A. 1999, Sol. Phys., 187, 89

Delannée, C., \& Aulanier, G. 1999, Sol. Phys., 190, 107

Foley, C. R., Harra, L. K., Culhane, J. L., \& Mason, K. O. 2001, ApJ, 560, L91

Foley, C. R., Harra, L. K., Culhane, J. L., et al. 2002, in MultiWavelength Observations of Coronal Structure and Dynamics, Yohkoh 10th Anniversary Meeting, ed. P. C. H. Martens, \& D. Cauffman, COSPAR Colloquia Ser., 13

Handy, B. N., Acton, L. W., Kankelborg, C. C., et al. 1999, Sol. Phys., 187, 229

Pike, C. D., \& Mason, H. 2002, Sol. Phys., 206, 359

Thompson, B. J., Gurman, J. B., Neupert, W. M., et al. 1999, ApJ, 517, L151 